\title{
Advances of Zero Flow Simulation of Air Conditioning Systems using Modelica
}

\author{
Pieter Dermont $^{1} \quad$ Dirk Limperich $^{2} \quad$ Johan Windahl $^{1} \quad$ Katrin Prölss $^{1} \quad$ Carsten Kübler $^{3}$ \\ ${ }^{1}$ Modelon AB, Sweden, \{pieter.dermont, johan.windahl, katrin.prolss\}@modelon.com \\ ${ }^{2}$ Daimler AG, Germany, \{dirk.limperich\}@daimler.com \\ ${ }^{3}$ TWT GmbH, Germany, \{carsten.kuebler\} atwt-gmbh.de
}

\begin{abstract}
This paper describes recent advances in simulation of zero flow conditions based on work with Daimler using the Air Conditioning Library from Modelon. The Air Conditioning Library is based on the open standard modelling language Modelica. Simulating refrigerant loops at (near) zero flow for large vapor compression cycles is challenging, due to the fast dynamics in the model under those conditions that drastically reduce the step size of the solver. Findings on solver selection and pressure drop correlations are presented. An approach to improve zero flow simulation based on a systematic analysis of heat transfer coefficients is suggested and demonstrated to increase simulation robustness under (near) zero flow conditions.
\end{abstract}

Keywords: air conditioning systems, zero flow, 1-D fluid modelling, dynamic simulation, numerical issues

\section{Introduction}

Operating modes with low and zero refrigerant mass flow rates in air-conditioning refrigeration systems have gained significance in the past years. Additional consumers of cooling power, such as batteries, evaporators for multi-zone air cooling or other integrated parts of the thermal management system have become more common.

Naturally, not all branches of the refrigeration cycle are active at all times but may be switched off during given operation modes. In addition, for the purpose of complete vehicle simulation, the entire cycle may be switched off at any time. The refrigeration system model is expected to handle these conditions smoothly and efficiently.

Characteristic for air conditioning cycles is the small total volume, often less than 1 [1]. In addition, the cycle traverses the two-phase dome, and as a consequence a wide and rapidly varying set of fluid properties.

\subsection{Thermofluid Modelling and the Air Con- ditioning Library}

Thermofluid models in Modelica are 1-D models which are effectively a string of control volumes and control surfaces (referred to as volume and flow components respectively in the Modelica community). Control volumes account for the conservation of energy and conservation, whereas the control surfaces incorporate the conservation of momentum (Figure 1). (Tummescheit, 2002) explains this concept in detail.

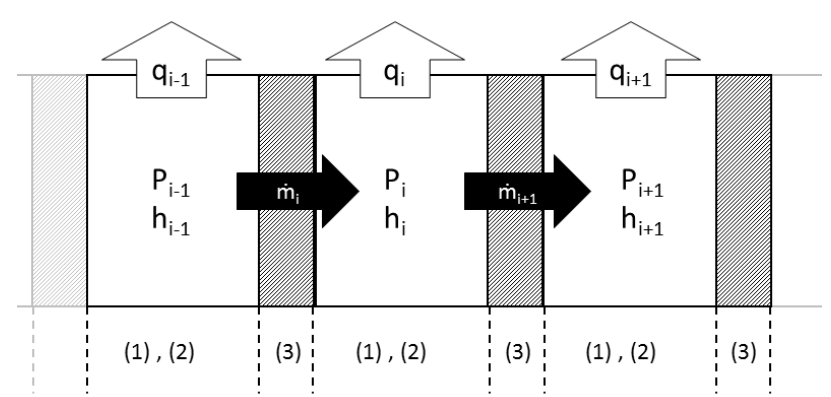

Figure 1. The balance equations. Conservation of mass (1) and energy (2) occurring in the control volume and the conservation of momentum (3) defined across an control surface.

In the Air Conditioning Library the models are discretized using an upwind discretization scheme, intended for (strong) convective flows with suppressed diffusion effects.

Both the above concepts are essential in this article and will be referred to at a later stage.

The Air Conditioning Library contains a set of predefined components focused on air conditioning applications and is developed by Modelon AB. It is currently mostly used by automotive OEM's and suppliers (Tummescheit et al., 2005).

\subsection{Test Models}

All investigations in the paper are based on a large system model, provided by Daimler. The system uses 
R134a refrigerant and contains 2 evaporator branches as depicted in Figure 2. Five use cases of the system model were described by Daimler:

\section{Normal operation}

2. Evaporator branch \#1 shutdown

3. Evaporator branch \#2 shutdown

4. Compressor shutdown through reduction of positive displacement volume. A complete simulation contains three shut-down - start-up cycles.

5. Compressor shutdown through reduction of shaft speed. Only one shut-down is performed and the system remains at rest for the rest of the simulation.

At the beginning of the investigation use cases 4-5 experienced significant increase in CPU time right after compressor shut-down, which prevented the simulation to complete within a reasonable time frame.

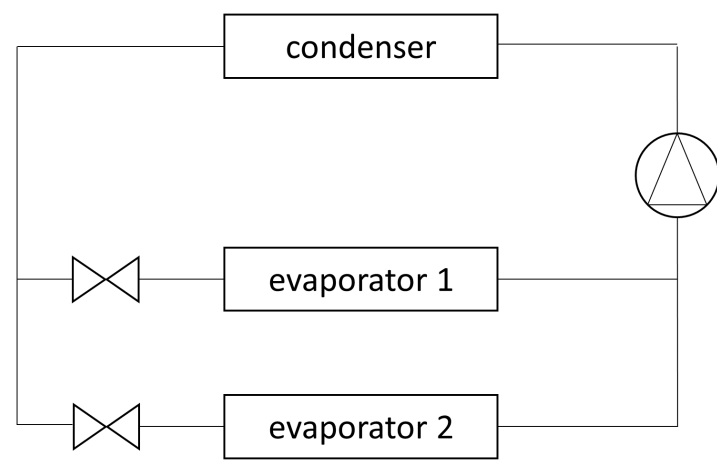

Figure 2. Schematic representation of the refrigeration system

\subsection{The Zero Flow Problem}

Close to zero flow, some oscillations appear due to the fast dynamics in the model. These dynamics are caused by the mathematical description that at nominal flow rates has reasonable time constants, but at low flow rates become very small. Hence, the solver reduces drastically its step size. A reduced solver step size during a prolonged period results in a dramatic increase in CPU time which makes it infeasible to compute the model within a reasonable time.

The fast dynamics can be observed in the simulation results by oscillations of a given set of variables, in particular the mass flow as is illustrated in Figure 3.

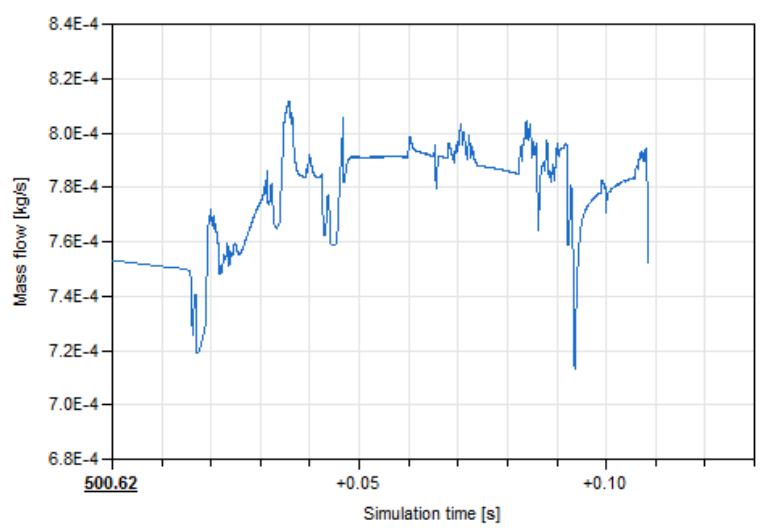

Figure 3. Oscillations in mass flow are a typical symptom of the zero flow problem.

The (simulation time, CPU time) graph is used repeatedly throughout this paper, as an illustration the progress the solver is making. The gradient of the graphs is an indication of the progress of the solver. Figure 4 shows the the graph for use case 4 before any improvements to the model, where the vertical graph gives a clear indication of the zero-flow problem. 3600 [s] is considered the limit of a reasonable simulation time. All simulations are automatically stopped at $3600[\mathrm{~s}]$ - a reasonable computation time for a system with approximately 270 continuous time states and many transients.

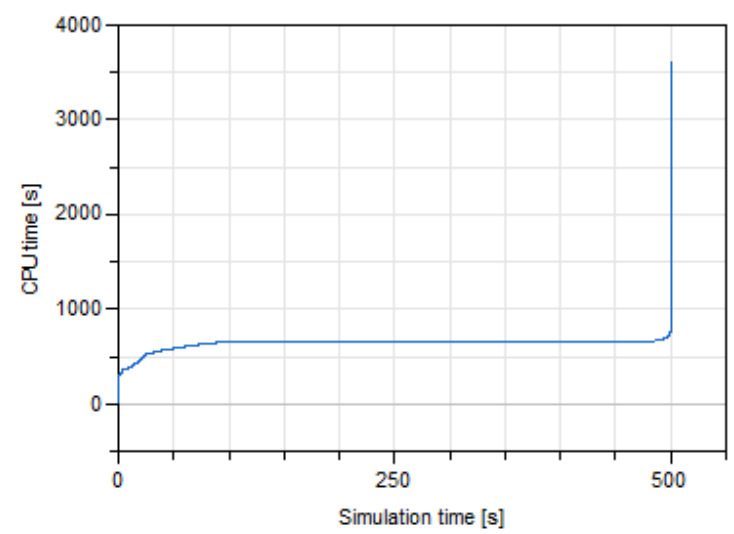

Figure 4. This graphs shows CPU time as a function of simulation time for use case 4. The vertical line at $\mathrm{t}=500$ [s] indicates the zero flow problem.

\section{Pressure Drop Correlation Regu- larization}

The pressure drop correlation presents in essence the relationship between pressure drop and mass flow, which is approximately a quadratic function (1) :

$$
\dot{m}=f(\triangle P) \sim \sqrt{\triangle P}
$$




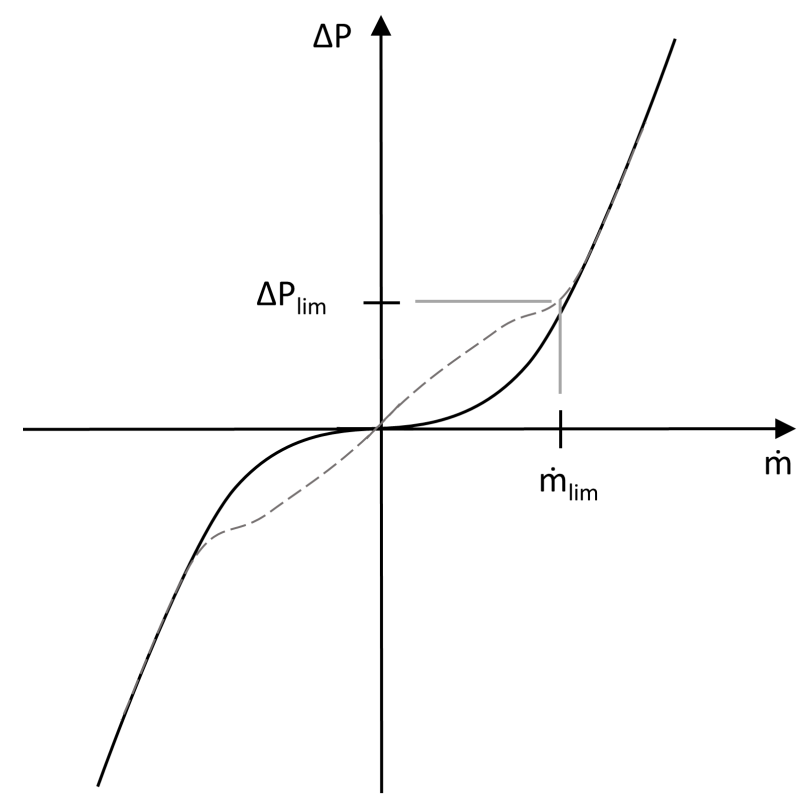

Figure 5. A regularized pressure drop

Note that the derivative of this function approaches zero when approaching a zero mass flow. This will disrupt the search algorithm as very small pressure differences correspond to very large variations in mass flow, causing the solver to jump between different solutions which are beyond solver tolerance. Regularized pressure drop correlations address this issue by increasing the derivative within a user specified region (Figure 5) (Tummescheit, 2002) . All pressure drop correlations in the Air Conditioning Library can be regularized with a parameter.

Operation point based pressure drop correlations, as opposed to geometry based pressure drop correlations where the pressure drop is specified by thermodynamic properties and the pipe geometry, allow the user to specify the nominal mass flow and pressure drop to define the pipe's behaviour. Note that depending on how the user sets the nominal mass flow and pressure drop, the correlation will be less or more sensitive to zero flow issues. A small pressure drop for a large mass flow will increase the region in which the derivative of the pressure drop mass flow function is beyond solver tolerance. It is thus beneficial to lump small pressure drops into one larger pressure loss.

A regularized pressure drop correlation is a necessary requirement for a complex thermo-fluid model to compute under zero flow conditions.

\section{Solver selection}

Using an appropriate solver is critical to ensure fast convergence of models that experience (near) zero flow operation. Three popular solvers are integrated in the simulation environment Dymola: Dassl, Lsodar, RadauII.
The former two are multi-step algorithms whereas the latter one is a one-step algorithm. One-step algorithms are more efficient at handling stiff problem formulations, which is the case for a (near) zero flow simulation, therefore RaduaII is the preferred solver.

A solver tolerance of $10 \mathrm{e}-6$ was used in Dymola 2016FD01.

\section{Heat Transfer To Control Volumes at Zero Flow}

As explained in Section 1.1, each control volume has an associated energy balance. For heat exchangers, a large contributor to the overall energy balance of the two phase channel is the heat transfer from or to the secondary channel governed by the heat transfer coefficient correlation.

\subsection{Observations}

In use case 4, oscillations are localised in an evaporator component. One can observe significant oscillations in mass flow and large oscillations in two phase fractions, in the control volumes located within the heat exchanger model. To address the problem, all heat transfer correlations are replaced by a constant $\left(\alpha=1500\left[\mathrm{~W} / \mathrm{m}^{2} \mathrm{~K}\right]\right)$ to investigate a potential improvement in the simulation progress. The result is remarkable; it does not improve the simulation at zero-flow, on the contrary, it reduces its robustness. The oscillations for this case occur for the control volumes located at the boundaries of the heat exchanger models.

Based on these observations, the fast dynamics are attributed to the (large) difference in heat transfer coefficients between adjacent control volumes:

1. Caused by to the difference in heat transfer between one-phase and two-phase flow, as is observed for use case 4.

2. Caused by the difference in heat transfer between heat exchangers and adiabatic pipe, as is observed when the heat transfer coefficient is set constant, i.e. without any mass flow dependency.

These hypotheses are strengthened by the result of a simulation run where all heat transfer coefficient correlations are replaced by a function for which the heat transfer coefficient is linearly dependent of the mass flow. The heat transfer coefficient is zero for zero mass flow and takes a given value at nominal flow rate (e.g. $\alpha=1500\left[\mathrm{~W} / \mathrm{m}^{2} \mathrm{~K}\right]$ ). Consequently, at zero-flow conditions no difference in heat transfer coefficient between adjacent control volumes exists, neither due to different phases nor at the interface heat exchanger - adiabatic pipe. Using this substitute correlation, all use cases compute effortlessly to the end. 


\subsection{Reduce the Difference in Heat Transfer Coefficient Between One-Phase and Two- Phase at Zero-Flow}

To accommodate for observation 1, heat transfer coefficients of one-phase and two-phase should converge to identical values at zero flow (Figure 6). Key points that were considered are:

- What value should the heat transfer coefficient converge to for zero flow? Since all two-phase heat transfer coefficient correlations that are used in this context are not valid at low flow rates but the onephase heat transfer coefficient correlations is, it is judicious to let the former converge towards the latter. However, its actual value is likely influenced by the droplets and bubbles.

- At what mass flow should such an transition begin? Two options are considered to set the transition threshold. In this section, (a fraction) of the nominal mass flow rate is used as limit. In the next section we explore the possibilities of using the Reynolds number instead.

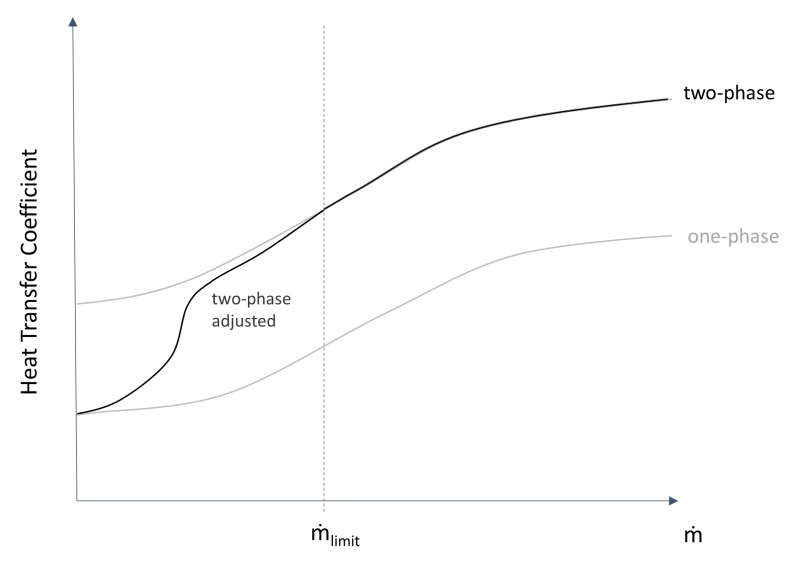

Figure 6. The heat transfer coefficient for one- and two-phase flow in function of the mass flow. To address the zero flow problem, the coefficient is smoothly interpolated between different phases

Note that this effectively not only addresses observation 1 but also applies to observation 2, since the heat transfer coefficient for one-phase flow is generally lower than for two-phase flow. This in turn decreases the difference between the heat transfer coefficients between control volumes located at the border of a heat exchanger and an adiabatic pipe.
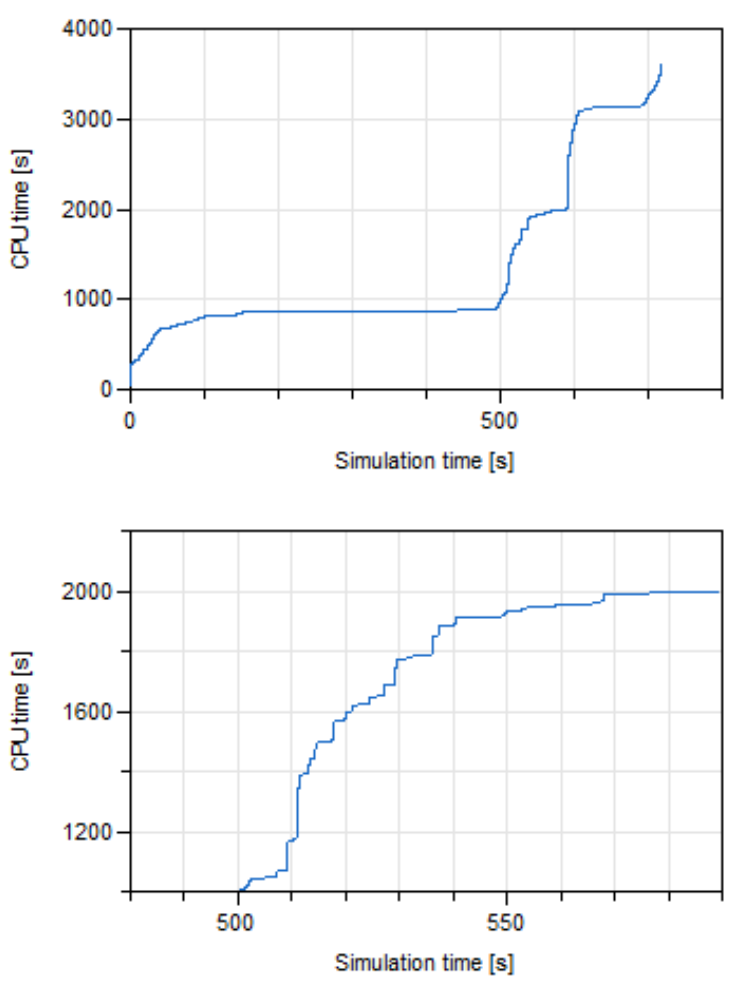

Figure 7. This graphs shows CPU time as a function of simulation time for use case 4, with smoothing based on mass flow. At 500 [s] the first compressor shut-down occurs. The simulation is stopped after 3600[s] CPU time, which allows for one complete compressor restart and another shut-down.

Performing this change proved to be highly beneficial to improve zero-flow behaviour. The simulation continues to make progress where it previously got stuck, as is demonstrated in Figure 7. The initial transients take approximately 800 [s] CPU time. Subsequently there a is plateau up to 500 [s] simulation time, where the cycle reaches steady state operation and the solver makes fast progress. The transients for cycle shut-down and restart are located between $500-600$ [s] simulation time and take approximately 1600 [s] CPU time, which is considered reasonable given the cost of the initial transient. The plateau located at approximately 600-700 [s] simulation time indicates a fast solver progress and coincides with normal refrigerant cycle operation.

\subsection{Filtering the Heat Transfer Coefficient}

While complete compressor shut-down and restart can be achieved without stalling the simulation, distinct steps in the (simulation time, CPU time) graph can still be observed (see Figure 7). These steps are caused by small variations of the two-phase fraction in a control volume, in its turn causing large variation in heat transfer coefficient between adjacent control volumes. The two-phase fraction indicates what the ratio of one-phase and twophase within a control volume is. The fast and local variations of heat transfer coefficient contribute to density 
variations and thus oscillating flow. A first order filter is applied to the heat transfer coefficient, with time constant $\tau$, as illustrated in Equation (2). Note that $F_{\text {user }}$ and $y$ are a calibration factor and the interpolation factor described in Section 4.2 respectively. This introduces an additional state in each control volume, which in principle will increase simulation time.

$$
\frac{d \alpha_{i}}{d t}=\frac{-\alpha_{i}+F_{u s e r}\left[y_{i} \alpha_{i}^{2 p h}+\left(1.0-y_{i}\right) \alpha_{i}^{1 p h}\right]}{\tau}
$$

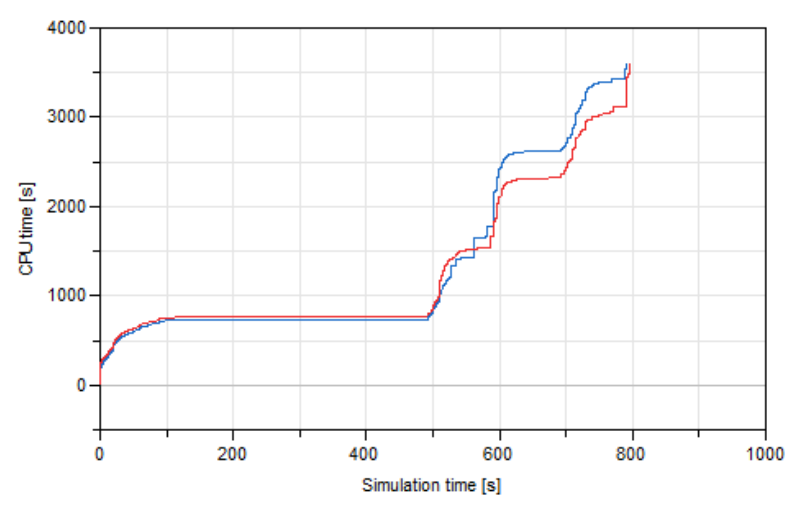

Figure 8. This graphs shows CPU time as a function of simulation time in compressor shut-down (case 4) using mass flow smoothing and a filter in heat transfer correlation. At 500 [s] the first compressor shut-down occurs. In this image, one can clearly see the different consecutive compressor shut-downs, 2-3 are achieved within 3600 [s] simulation time. The blue line corresponds to $\tau=0.01$, and the red $\tau=0.001$.

Applying the filter has a recognizable effect on the simulation robustness. In Figure 8, within 3600[s] of CPU time, between 1-2 compressor shut-downs are achieved. The value of the time constant was varied $\tau=10^{-2}-10^{-6}[1 / s]$; the concept was beneficial in all cases but no universally optimal value could be determined.

\subsection{Reynolds Number Smoothing}

In order to achieve a more physically correct result, it was suggested to set a Reynolds number as threshold value for the two-phase heat transfer coefficient to converge towards the one-phase heat transfer coefficient when approaching zero mass flow. The Reynolds number is a more meaningful measurement, independent of the pipe geometry and as it is an indication of the flow regime, a metric often used to describe the validity of heat transfer correlations.

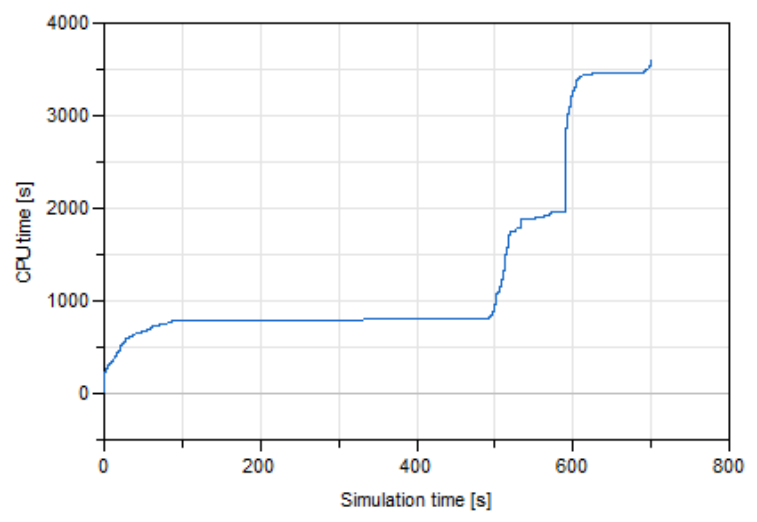

Figure 9. This graphs shows CPU time as a function of simulation time in compressor shut-down (case 1) using Reynolds based smoothing and a filter in heat transfer correlation. At 500 [s] the first compressor shut-down occurs. A complete compressor shut-down and restart cycle is completed within 3600 [s] CPU time.

As default value, $R e=3000[-]$ is used, located in the transition between laminar $(R e<2300[-])$ and turbulent flow $(R e>4000[-])$. Using this threshold the simulation proceeds steadily, however at a slower rate as the previously used nominal mass flow threshold (Figure 9).

\subsection{Physical Behaviour at Zero Flow}

The suggestions for improved zero flow robustness described above rely on altering the heat transfer coefficients from existing heat transfer coefficient correlations when approaching zero flow. This has consequences on the model behaviour at zero flow but not in the nominal operation range, provided the threshold value of the mass flow or $R e$ is correctly set. If the time constant $\tau$ is set sufficiently low, it has no significant impact on the result in nominal operation mode.

Topics for future consideration are:

1. What is the value of the heat transfer coefficient at zero flow? All two-phase correlations implemented in the Air Conditioning Library cease to be valid at $R e=3000[-]$. One-phase correlations however, remain valid to a much lower Reynolds number well into the laminar region. It appears therefore justified to converge towards the one-phase heat transfer coefficient correlation. However, for a more accurate value an advection correlation would need to be included in the overall heat transfer coefficient calculation.

2. What is the effect of gravity on the system? The models in the Air Conditioning Library do not take into account the effect of gravity nor introduce a slip factor between phases. At low flow rates, phase separation will occur. 
3. How do these two items above interact? If we assume that at zero flow, after the system has come to rest, the channels are filled up with either liquid or vapour only, the one-phase correlation is more likely to mimic the physics better. However, boiling is likely to occur in given sections of the cycle.

\section{Considerations for Future Work}

The discretization scheme currently implemented only accounts for convective flow as previously specified. At zero flow, the energy balances of adjacent control volumes are not linked and the only relationship between adjacent control volumes is the momentum balance. By altering the heat transfer coefficient, the need for linking the energy balances is reduced, as the the energy accumulation/dissipation is more uniform.

Instead, one could imagine creating a thermal link between adjacent control volumes. Diffusion-like discretization schemes can be included in the energy conservation equations of each control volume. This has been done previously however did not yield satisfactory results. While diffusion is beneficial to the zero flow problems, it cannot offset the very large energy accumulation differences between adjacent control volumes that have very different heat transfer coefficients, unless the diffusion factors are unreasonably high.

And alternative method to link the energy balances of the control volumes is to define a discretized (metal) wall along side the thermo-fluid model, through which the control volumes can indirectly interact. The additional states will increase simulation time under nominal conditions but may likely improve model robustness under zero flow conditions.

\section{Conclusion}

The approach for improved zero flow behaviour of detailed air conditioning system models uses the heat transfer coefficient correlations which prescribe the heat transfer from the refrigerant control volumes to the secondary side. The implementation requires that the value of these coefficients for adjacent control volumes approach each one another for (near) zero flow simulation. In refrigeration simulation, the two natural occurrences of large variations of heat transfer coefficients exist between adiabatic pipes and heat exchangers and between one- and two-phase coefficients within heat exchangers. An approach to numerically smooth these transitions based on nominal mass flow and Reynolds number for the latter suggested. The approach is tested with large system models and demonstrated to increase simulation robustness under (near) zero flow conditions. Systems simulations during which the compressor is repeatedly shut-down previously got stuck but now run to the end.

\section{References}

Hubertus Tummescheit. Design and implementation of objectoriented model libraries using modelica. $\mathrm{PhD}$ thesis, Lund University, 2002.

Hubertus Tummescheit, Jonas Eborn, and Katrin Prolss. Airconditioning-a modelica library for dynamic simulation of ac systems. In 4th International Modelica Conference, 2005. 